Supporting Information for:

\title{
Biodegradable Polymer Microparticles with Tunable Shapes and Surface Textures for Enhancement of Dendritic Cells Maturation
}

Mubashir Hussain", Jun Xie", Ke Wang, Hua Wang, Zhengping Tan, Qianqian Liu, Zhen Geng, Khurram Shezad, Laila Noureen, Hao Jiang, Jiangping Xu*, Lianbin Zhang and Jintao Zhu*

Key Laboratory of Materials Chemistry for Energy Conversion and Storage (HUST), Ministry of

Education, School of Chemistry and Chemical Engineering, Huazhong University of Science and Technology (HUST), Wuhan 430074, China

*Corresponding authors. E-mail: jiangpingxu@hust.edu.cn (J.X)

jtzhu@mail.hust.edu.cn (J.Z);

\#These authors contributed equally to this paper. 


\section{Supporting figures:}
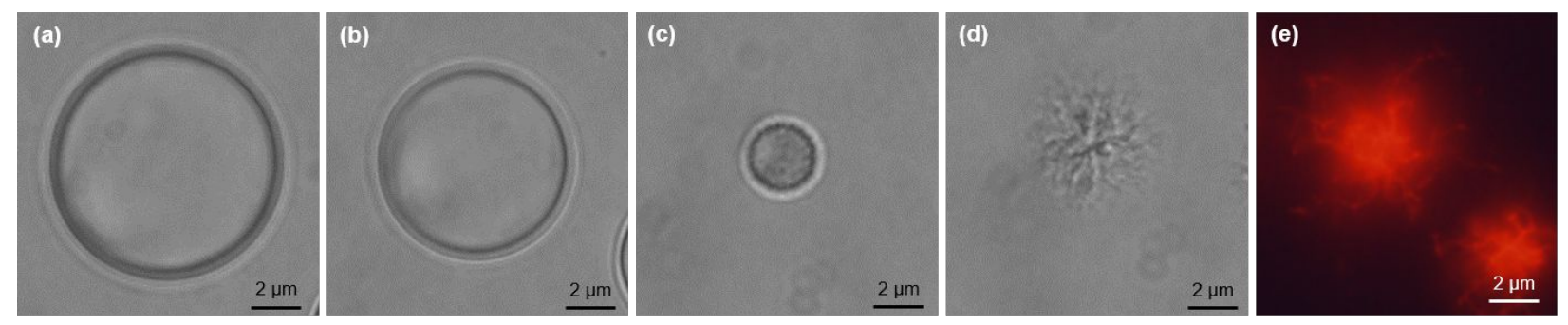

Solvent Evaporation

Figure S1. Optical and fluorescence microscopy images for the evolution of emulsion droplets of dichloromethane containing $\mathrm{PLA}_{28 \mathrm{k}}-b-\mathrm{PEG}_{5 \mathrm{k}}(10 \mathrm{mg} / \mathrm{mL})$. (a)-(c) As the evaporation of solvent proceeds, the droplet size decreases and the concentration of BCP increases. (d) The surface of the droplet does not remain smooth and gets fuzzy and then explodes, resulting in the formation of numerous radial fibers. Image (e) is the respective fluorescence microscopy photograph of particles with many fiber-like protrusions. 


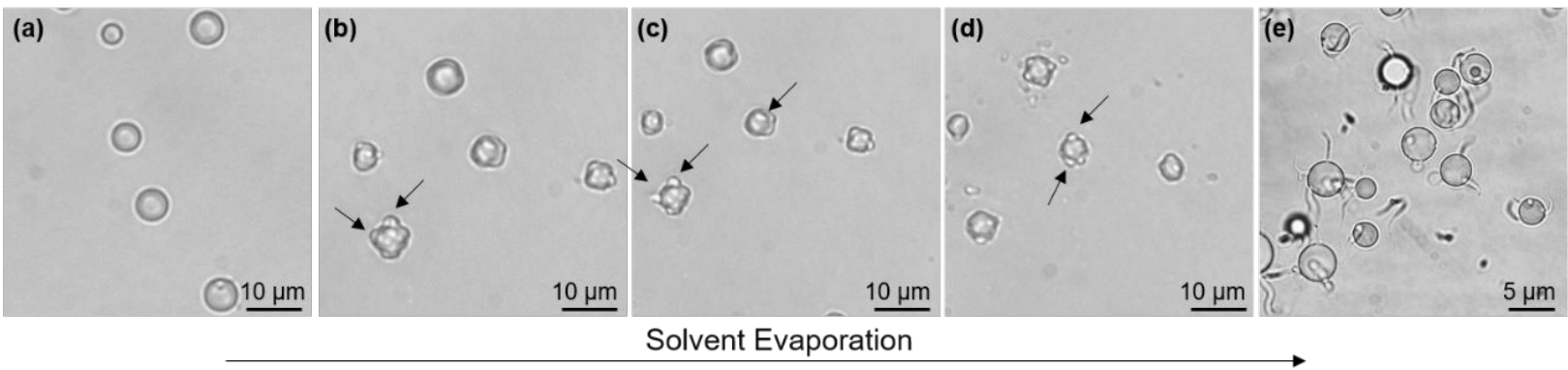

Figure S2. Optical microscopy images for the evolution of emulsion droplets of DCM containing PLGA $_{100 \mathrm{k}}$ and $\mathrm{PLA}_{16 \mathrm{k}}-b-\mathrm{PEG}_{5 \mathrm{k}}$ at a blend ratio of $1: 1$. With the extraction of DCM, small protrusions start to appear at the edges of droplets as pointed by arrows in the image (b)-(d). Length of protrusions increases as the evaporation of DCM. After complete removal of DCM, the morphology of the microparticles is frozen, and the tentacular microparticles can be obtained (e). 

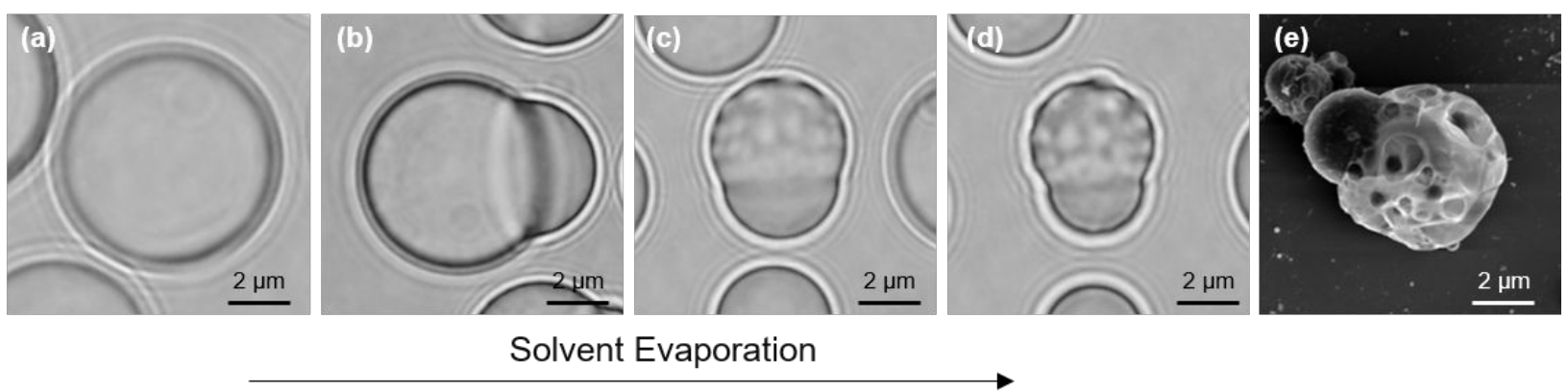

Solvent Evaporation

Figure S3. (a)-(d) Optical microscopy images of shrinking emulsion droplets containing PLGA Pook and $\mathrm{PLA}_{48 \mathrm{k}}-b-\mathrm{PEG}_{2 \mathrm{k}}$ blends (weight ratio 1:1). The blends were dissolved DCM and processed via microfluidics to produce uniformly-sized emulsion droplets in aqueous PVA (a). Upon extraction of DCM, spherical emulsion droplets transform into oval droplets as represented in image (b) and finally turn into Janus microparticles with rough hemisphere comprising of $\mathrm{PLA}_{48 \mathrm{k}}-b-\mathrm{PEG}_{2 \mathrm{k}}$ and smooth hemisphere corresponding to PLGA $_{100 k}(\mathrm{c})$, (d). Image (e) represents SEM image of a Janus microparticle. 

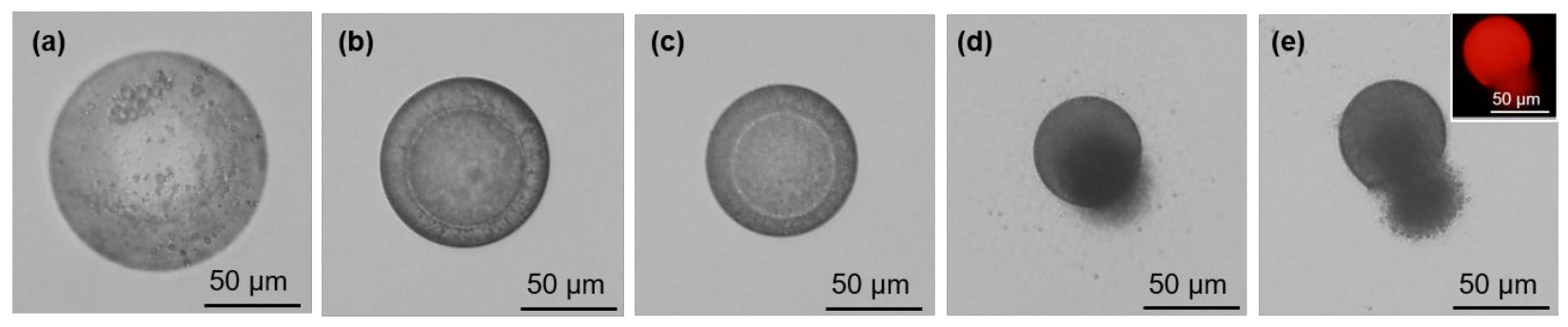

Figure S4. Optical microscopy images showing the formation of hollow microparticles obtained by blending $\mathrm{PLGA}_{100 \mathrm{k}}$ and $\mathrm{PLA}_{16 \mathrm{k}}-b-\mathrm{PEG}_{5 \mathrm{k}}$ at $70: 30$ in trichloromethane. Image (a) shows an emulsion droplet of the blend. Phase separation starts to take place between two phases (b)-(c). $\mathrm{PLA}_{16 \mathrm{k}}-b-\mathrm{PEG}_{5 \mathrm{k}}$ is released in the surrounding aqueous medium (d)-(e). Inset in image (e) is the representative fluorescence photograph. 


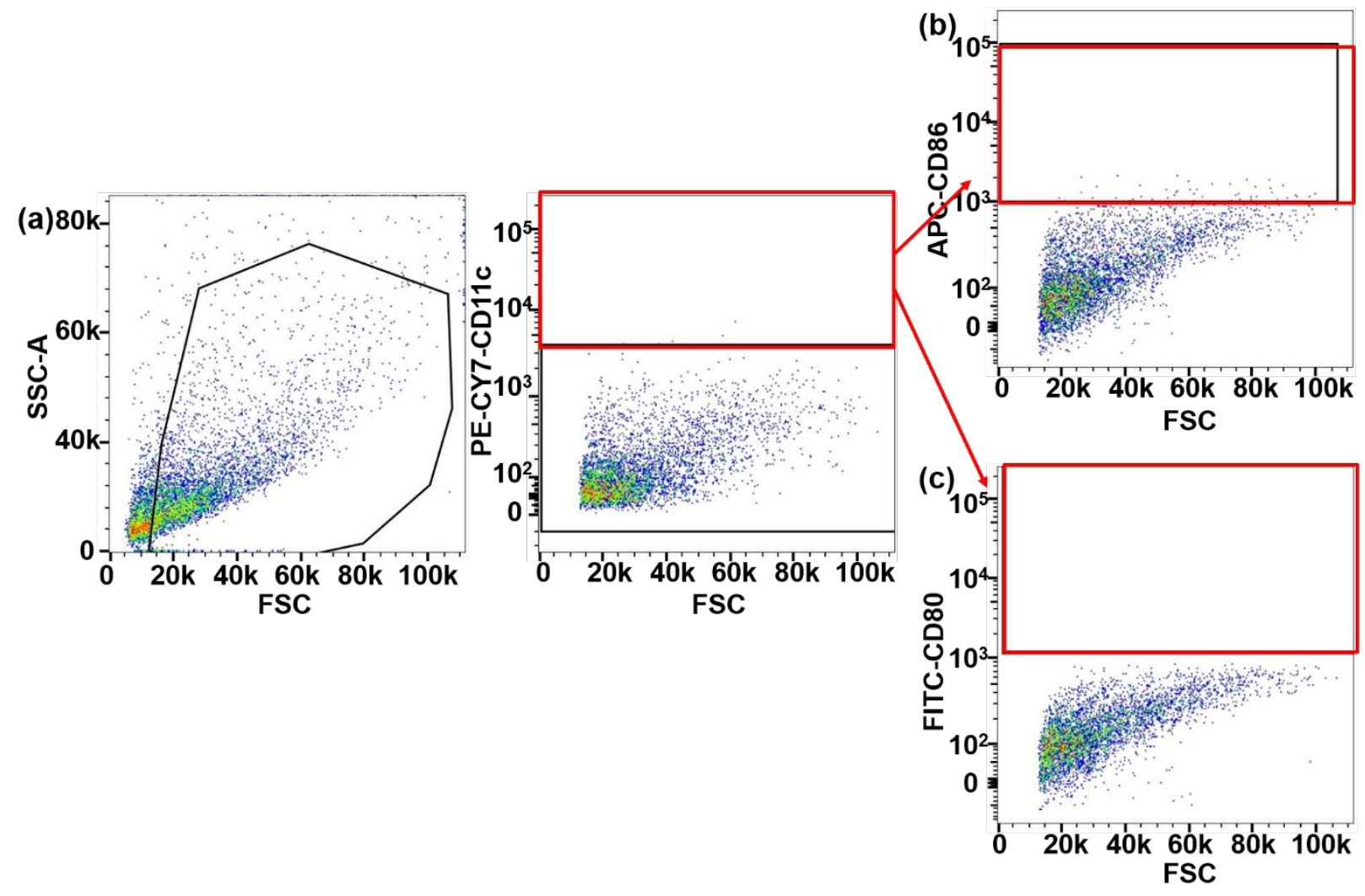

Figure S5. Flow cytometry analysis of the expression of CD86 and CD80 on the DCs stimulated by the different microparticles after co-culturing for $24 \mathrm{~h}$. Corresponding isotype controls of (a) stained with PE-Cy7-CD11c for dendritic cells, (b) stained with APC for CD86 and (c) stained with FITC for CD80. 

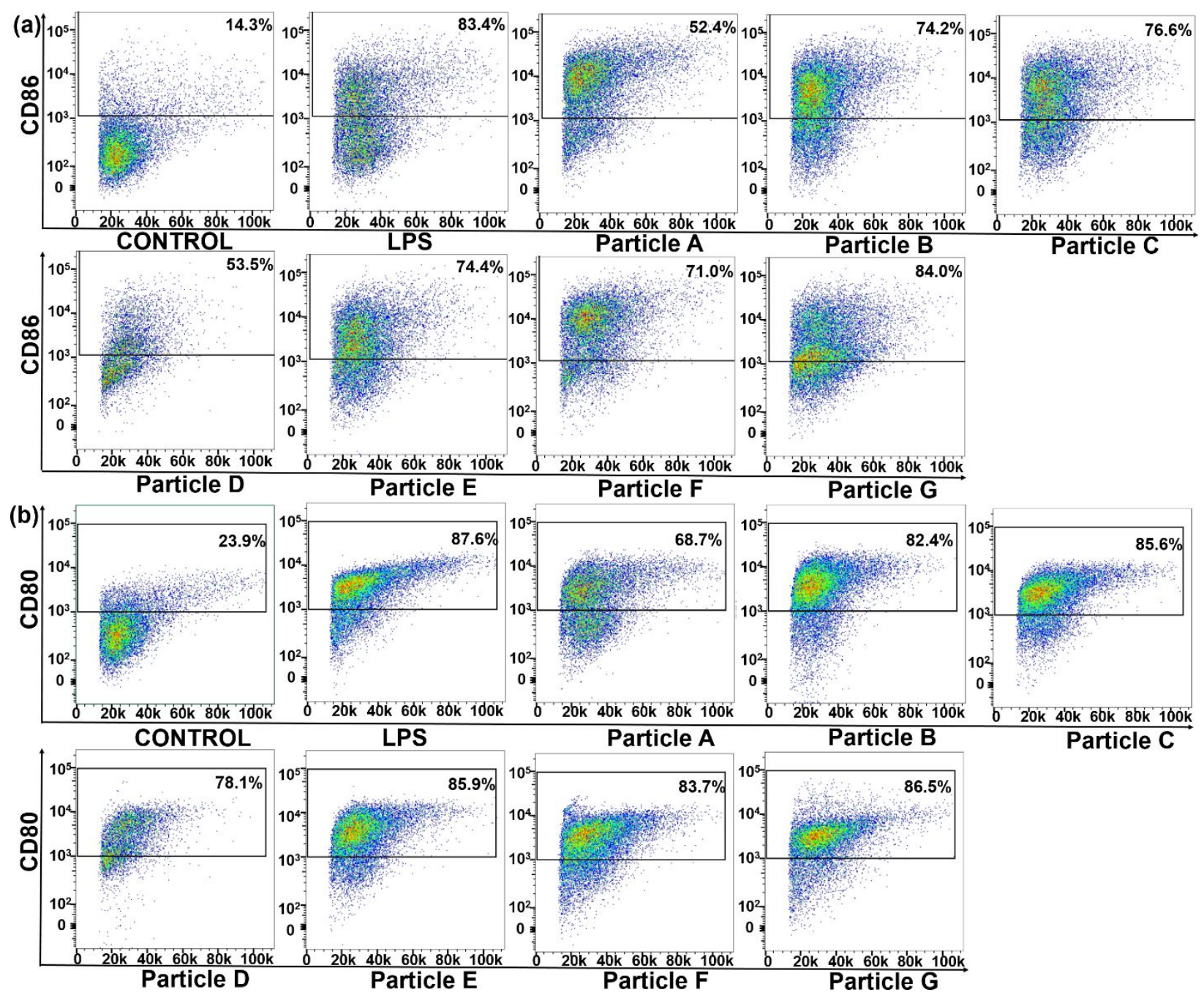

Figure S6. Flow cytometry analysis of the expression of (a) CD86 and (b) CD80 on the DCs stimulated by the microparticles with varying morphologies and surface textures (Particle A, Particle B, Particle C, Particle D, Particle E, Particle F, Particle G) after co-culturing for 24 h. PBS was used as negative control while LPS was applied as positive control for all experiments. 


\section{Supporting Movies:}

Movie S1. Real time video showing the evolution of emulsion droplets containing PLA $16 \mathrm{k}-b-\mathrm{PEG}_{5 \mathrm{k}}$ in oil phase. The droplets were generated by using single emulsion glass micro-capillary device.

Movie S2. Real time video showing the formation of tentacular microparticles by dissolving $\mathrm{PLA}_{16 \mathrm{k}}-b-\mathrm{PEG}_{5 \mathrm{k}}$ and PLGA $\mathrm{P}_{100 \mathrm{k}}$ at $1: 1$ in dichloromethane. Aqueous PVA (5 mg/mL) was applied as the continuous phase.

Movie S3. Real time video showing the formation of hollow hemispherical microparticles by dissolving $\mathrm{PLA}_{16 \mathrm{k}}-b-\mathrm{PEG}_{5 \mathrm{k}}$ and $\mathrm{PLGA}_{100 \mathrm{k}}$ at $1: 1$ in trichloromethane. Aqueous PVA $(5 \mathrm{mg} / \mathrm{mL})$ was applied as the continuous phase. 